\title{
Implications of transitioning to a resident night float system in neurosurgery: mortality, length of stay, and resident experience
}

\author{
Matthew C. Davis, MD, ${ }^{1}$ Elizabeth N. Kuhn, MD, ${ }^{1}$ Bonita S. Agee, PhD, MPH, ${ }^{1}$ Robert A. Oster, PhD, ${ }^{2}$ \\ and James M. Markert, MD, MPH ${ }^{1}$ \\ 1Department of Neurosurgery and ${ }^{2}$ Center for Clinical and Translational Sciences, University of Alabama at Birmingham, \\ Alabama
}

OBJECTIVE Many neurosurgical training programs have moved from a 24-hour resident call system to a night float system, but the impact on outcomes is unclear. Here, the authors compare length of stay (LOS) for neurosurgical patients admitted before and after initiation of a night float system at a tertiary care training hospital.

METHODS The neurosurgical residency at the University of Alabama at Birmingham transitioned from 24-hour call to a night float resident coverage system in July 2013. In this cohort study, all patients admitted to the neurosurgical service for 1 year before and 1 year after this transition were compared with respect to hospital and ICU LOSs, adjusted for potential confounders.

RESULTS A total of 4619 patients were included. In the initial bivariate analysis, night float was associated with increased ICU LOS $(p=0.032)$ and no change in overall LOS $(p=0.65)$. However, coincident with the transition to a night float system was an increased frequency of resident service transitions, which were highly associated with hospital LOS $(p<0.01)$ and ICU LOS $(p<0.01)$. After adjusting for resident service transitions, initiation of the night float system was associated with decreased hospital LOS ( $p=0.047)$ and no change in ICU LOS $(p=0.35)$.

CONCLUSIONS This study suggests that a dedicated night float resident may improve night-to-night continuity of care and decrease hospital LOS, but caution must be exercised when initiation of night float results in increased resident service transitions.

https://thejns.org/doi/abs/10.3171/2016.5.JNS152585

KEY WORDS length of stay; neurosurgery; patient handoff; patient outcome assessment

I N 2003, the Accreditation Council for Graduate Medical Education (ACGME) imposed 80-hour work week restrictions on all residency programs in the United States. ${ }^{2}$ Even though neurosurgical training programs may apply for an 88-hour work week exemption, ${ }^{6}$ providing 24-hour in-house resident coverage while meeting strict work hour requirements often proves difficult, particularly for programs with smaller resident corps. In partial response, many training programs have moved from a 24hour resident call system to a night float system. ${ }^{34} \mathrm{~A}$ night float resident typically provides in-house overnight coverage 5 or 6 nights per week, with other residents taking 24-hour call shifts on the remaining days. These residents provide night-to-night continuity of care for neurosurgical patients, see and manage consults, and perform emergent bedside procedures. Residents usually spend from 1-3 consecutive months on a night float rotation, with the total duration of night float for each resident dependent on the number of residents and number of hospitals requiring inhouse coverage.

Critics of the night float system have expressed concern over what they view as weaker clinical and operative training due to "lost" months spent on night float rotations, increased number of resident handoffs with resultant potential for medical errors, and risk of engendering a shift-work mentality among neurosurgical residents. ${ }^{20}$ Irrespective of the underlying argument, in this era of training, the ability of neurosurgical residents to

ABBREVIATIONS ACGME = Accreditation Council for Graduate Medical Education; ANCOVA $=$ analysis of covariance; $D R G=$ diagnosis-related group; $L O S=$ length of stay; $P G Y=$ postgraduate year; $U A B=$ University of Alabama at Birmingham.

SUBMITTED November 7, 2015. ACCEPTED May 13, 2016.

INCLUDE WHEN CITING Published online July 8, 2016; DOI: 10.3171/2016.5.JNS152585. 
provide quality care while receiving adequate training has been scrutinized. ${ }^{20,23}$ Following implementation of duty-hour restrictions, multiple studies have reported increased postoperative complications and hospital charges in neurosurgical patients, with no improvement in outcomes. 7,821,32 Additionally, the ACGME mandates that residents have a minimum break period between shifts, which necessitates an increased frequency of handoffs and fragments continuity of care. ${ }^{24}$

The influence of a night float system on patient outcomes in neurosurgery has never been rigorously evaluated. Here, we compare hospital and neurosurgical ICU lengths of stay (LOSs) for neurosurgical patients admitted before and after initiation of a night float system at an academic tertiary care training hospital, adjusting for potential confounders.

\section{Methods}

\section{Patient Selection and Sample Size Determination}

The neurosurgical training program at the University of Alabama at Birmingham (UAB) transitioned from a 24-hour call to a night float resident coverage system in July 2013. All patients admitted to the neurosurgical service for 1 calendar year before and after this transition were identified from billing records. Only patients for whom a neurosurgeon was the primary attending of record were included. Patients for whom neurosurgery was a consulting service only were excluded. Sample size was determined by the availability of complete resident call schedules, which were not preserved prior to July 2012. The follow-up period ended at hospital discharge. Investigators collecting data on outcomes of interest were blinded to presence/absence of exposure to the night float system as well as coincident resident service transition. Data were collected from the electronic medical record.

\section{Data Collection}

Data collected for this study included patient age, sex, comorbidities, hospital LOS, ICU LOS, coincident resident service transition, admission status (elective admission for surgery, admission through the emergency department, admission from clinic, transfer from outside hospital), admission unit (ICU, stepdown unit, floor), diagnosis-related group (DRG), discharge disposition (home, died, hospice, left against medical advice, another acute hospital, inpatient rehabilitation, skilled nursing facility, psychiatric hospital, other long-term hospital), whether the stay included a weekend, 30-day readmission rates, and mortality. We additionally collected overall LOS for the 5 most common DRGs for the past 7 years, the period for which accurate data were available. Data were collected retrospectively via chart review. The exposure of interest was the night float call system. The primary outcome of interest was overall LOS. ICU LOS and mortality were secondary outcomes in the initial study design. A service transition was defined as any point when a resident handed off coverage of a service for longer than a weekend. Transitions occurred when residents switched between attending services, during vacations, and other absences. These transitions refer to day-coverage residents only, as night float residents cover all patients on the neurosurgical service. Previous work demonstrated that resident service transitions were highly associated with LOS. ${ }^{26}$ Dates of service transitions were collected from resident service, meeting, and vacation schedules. Patients admitted for outpatient surgery who did not stay overnight were excluded due to no chance of exposure to the night float system.

\section{Resident Experience}

Operative case logs were obtained from the ACGM online data collection system for all postgraduate year (PGY) 2 through 6 residents at the UAB during the years under study. Reported duty hours were additionally collected for the same resident cohorts during the years under study. Resident perception on the overall utility of the night float system was assessed via questionnaire using a 5-point Likert scale, where 1 = significantly decreases/worsens, 2 $=$ somewhat decreases $/$ worsens, $3=$ no effect, $4=$ somewhat increases/improves, and $5=$ significantly increases/ improves. Residents were queried using an electronic survey sent to all 19 residents at our institution. Reminder emails were sent every 5-10 days until $100 \%$ response had been achieved. No incentive was provided to respondents. Responses were de-identified and kept anonymous.

\section{Bias}

The following steps were taken to address potential sources of bias. Investigators were blinded to exposure to night float and the occurrence of resident service transitions. A single large group, consisting of all neurosurgical patients admitted to UAB Hospital during the study period, was used for identification of both cohorts to limit selection bias. Recall bias was limited by collecting data solely from the electronic medical record rather than dependence on patient or provider memory.

\section{Statistical Analysis}

Patients admitted before initiation of night float $(\mathrm{n}=$ 2173) were compared with patients admitted after initiation of night float $(n=2446)$ with respect to the following: overall hospital LOS, ICU LOS, mortality, admission status, admission unit, age, sex, comorbidities, occurrence of resident service transition, discharge disposition, and 30-day readmission rates. There were no missing data. Chi-square tests (or Fisher's exact tests when assumptions were not met) were used to assess statistical significance of differences for categorical variables. Differences between continuous variables were compared using 2-sample independent t-tests. Analysis of covariance (ANCOVA) multivariate analysis was performed to determine the independent effect of introducing the night float system on the outcomes of interest. Only variables found to be significantly associated with the outcome of interest were included in the multivariate analysis. Furthermore, a matched-pairs design subset analysis was performed for 2 patient groups: patients undergoing cervical fusion (DRG codes 471-473) and patients undergoing pituitary surgery (DRG codes 614). These procedure subsets were selected as they were performed at high frequency and were likely to consist of similar patient populations. The exposure of 
interest was exposure to the night float system. Groups were matched by DRG, age (within 3 years), and sex. The outcome of interest was overall hospital LOS and ICU LOS. All statistical analyses were performed using JMP (version 11.0.0, SAS Institute Inc.).

Preanalysis data screening was performed to determine whether an ANCOVA was appropriate. Normality of distribution of LOS was confirmed using the KolmogorovSmirnov test. The relationship between the covariates and dependent variable was not influenced by the independent variable, thus confirming the homogeneity of slopes assumption. The assumptions of independence and normality were met. The assumption of homogeneity of variance was tested using Levene's test, and the results were not found to be significant ( $F$ ratio $=0.007 ; p=0.93$ ), indicating that the assumption of homogeneity of variance was met.

Post hoc linear regression analysis was performed for overall hospital LOS from 2008 through 2014 for the 5 most common admission DRG codes of patients included in the study, to assess for trends in LOS over an extended period of time. Matched pairs Student t-tests were performed for the above DRG codes for the 2 years immediately prior to initiation of night float. Finally, we assessed the distribution of the 5 most common admission DRG codes for pre-night float and night float years.

This study was approved by the Institutional Review Board at the University of Alabama at Birmingham. The manuscript was prepared following the STROBE (Strengthening the Reporting of Observational Studies in Epidemiology) checklist for cohort studies.

\section{Results \\ Study Population}

A total of 5497 patients met criteria for inclusion in the study; 878 patients were excluded as they underwent outpatient surgery and did not stay overnight. This left 4619 patients who were included in the final analysis. There were 2173 patients admitted before the initiation of night float, compared with 2446 patients admitted after the initiation of night float. Among the pre-night float group, the mean patient age was $55.0 \pm 16.1$ years (SD), and $51.1 \%$ were male. Among the night float group, the mean patient age was $54.9 \pm 15.6$ years and $49.3 \%$ were male. There was no statistically significant difference in age $(\mathrm{p}=0.8)$ or sex ( $p=0.2$ ) between the groups. Furthermore, there was no difference in admission status $(\mathrm{p}=0.08)$, admission unit $(\mathrm{p}=0.5)$, whether the hospital stay included a weekend ( $\mathrm{p}$ $=0.3$ ), or comorbidities (Table 1$)$.

\section{Resident Service Transitions}

In the pre-night float era, there were 253 service transitions (11.6\%), while there were 346 transitions in the night float era $(14.2 \%)$. The proportion of transitions was significantly higher in the night float era $(\mathrm{p}=0.01)$, and concomitant resident service transition was associated with prolonged hospital LOS $(p<0.01)$ and ICU LOS $(p<0.01)$. The magnitude of the observed effect of service transitions on length of ICU LOS ( $p=0.7)$ and overall hospital LOS $(p=0.8)$ was not significantly different between pre- and post-night float groups. Figure 1 demonstrates the changes to resident service coverage that accompanied moving to a night float system. Figure 2 demonstrates the day-to-day call structure in the pre-night float and night float eras.

\section{Overall Length of Stay}

The mean LOS in the pre-night float group was 5.2 days (95\% CI 4.8-5.5), while the mean LOS in the night float group was 5.1 days (95\% CI 4.7-5.4). Bivariate analysis using 2-sample independent Student t-tests indicated that there was no significant impact on overall LOS after initiation of night float ( $p=0.7$, Table 2). Adjusting for the increased number of service transitions in the night float era, there was a significant decrease in the overall LOS after the institution of night float $\left(\mathrm{R}^{2}=0.25\right.$, root-meansquare error $=7.05, \mathrm{p}=0.047$ ). To further characterize the effect of night float on LOS in the absence of resident service transition, we examined the subgroup of patients who did not have a resident service transition. Within this subgroup, the effect was consistent. The mean LOS in the pre-night float group was 3.7 days (95\% CI 3.5-3.9 days), while the mean LOS in the night float group was 3.4 days (95\% CI 3.2-3.6 days). Bivariate analysis using 2-sample independent $\mathrm{t}$-tests indicated that there was a significant decrease in overall LOS after the initiation of night float among those patients who had not experienced a resident service transition $(\mathrm{p}=0.01)$.

We performed additional post hoc linear regression analysis of overall hospital LOS from 2008 through 2014 for the 5 most common admission DRG codes for patients included in the study. We found a significant trend in decreased overall LOS during this period for DRG code 25 (intracranial procedure with major comorbidities/ complications, $\mathrm{R}^{2}=0.72, \mathrm{p}=0.02$ ), DRG 27 (intracranial procedure without comorbidities/complications or major comorbidities/complications, $\mathrm{R}^{2}=0.75, \mathrm{p}=0.01$ ), and

TABLE 1. Demographics and clinical variables of the cohorts*

\begin{tabular}{lccc}
\hline \multicolumn{1}{c}{ Variable } & $\begin{array}{c}\text { Night Float } \\
(\mathrm{n}=2446)\end{array}$ & $\begin{array}{c}\text { No Night Float } \\
(\mathrm{n}=2173)\end{array}$ & $\begin{array}{c}\mathrm{p} \\
\text { Value† }\end{array}$ \\
\hline Mean age in yrs \pm SD & $55.0 \pm 16.1$ & $54.9 \pm 15.6$ & 0.8 \\
\hline Male sex, \% & 51.1 & 49.3 & 0.2 \\
\hline Admission via ED & $443(18)$ & $439(20)$ & 0.08 \\
\hline Admission to neurosurgical ICU & $470(19)$ & $441(20)$ & 0.5 \\
\hline Elective admission for surgery & $1289(53)$ & $1110(51)$ & 0.09 \\
\hline $\begin{array}{l}\text { Hospital stay extending over } \\
\quad \text { weekend }\end{array}$ & $559(23)$ & $516(24)$ & 0.3 \\
\hline Atrial fibrillation & 114 & 118 & 0.7 \\
\hline Hypertension & 1128 & 1107 & 0.2 \\
\hline Type II diabetes mellitus & 339 & 308 & 0.6 \\
\hline Coronary artery disease & 71 & 59 & 0.5 \\
\hline Morbid obesity & 111 & 96 & 0.7 \\
\hline Tobacco use & 445 & 421 & 0.13 \\
\hline Congestive heart failure & 93 & 73 & 0.3 \\
\hline
\end{tabular}

* Values represent the number of patients (\%) unless noted otherwise.

$\dagger$ Student t-test was used for comparison of means; chi-square was used for comparison of proportions. 


\begin{tabular}{|c|c|c|c|c|c|c|c|c|c|}
\hline & \begin{tabular}{|c|} 
July \\
\end{tabular} & \begin{tabular}{|l|} 
August \\
\end{tabular} & \begin{tabular}{|l|} 
Sept \\
\end{tabular} & Oct & Nov & Dec & Jan & Feb & March \\
\hline Resident A & Attending $1 \& 4$ & Attending $1 \& 4$ & Attending $1 \& 4$ & Attending 2 & Attending 2 & Attending 2 & Attending 3 & Attending 3 & Attending 3 \\
\hline Resident B & Attending 3 & Attending 3 & Attending 3 & Attending $1 \& 4$ & Attending $1 \& 4$ & Attending $1 \& 4$ & Attending 2 & Attending 2 & Attending 2 \\
\hline Resident C & Attending 2 & Attending 2 & Attending 2 & Attending 3 & Attending 3 & Attending 3 & Attending $1 \& 4$ & Attending $1 \& 4$ & Attending $1 \& 4$ \\
\hline \multicolumn{10}{|l|}{ Night Float } \\
\hline & July & August & Sept & Oct & Nov & Dec & Jan & Feb & March \\
\hline Resident A & Attending $1 \& 4$ & Attending $2 \& 3$ & Night Float & Attending $1 \& 4$ & Attending $2 \& 3$ & Night Float & Attending $1 \& 4$ & Attending $2 \& 3$ & Night Float \\
\hline Resident B & \begin{tabular}{|l|} 
Night Float \\
\end{tabular} & Attending $1 \& 4$ & Attending $2 \& 3$ & \begin{tabular}{|l|} 
Night Float \\
\end{tabular} & Attending $1 \& 4$ & Attending $2 \& 3$ & \begin{tabular}{|l|} 
Night Float \\
\end{tabular} & Attending $1 \& 4$ & Attending $2 \& 3$ \\
\hline Resident C & Attending $2 \& 3$ & Night Float & Attending 1\&4 & Attending $2 \& 3$ & \begin{tabular}{|l|} 
Night Float \\
\end{tabular} & Attending $1 \& 4$ & Attending $2 \& 3$ & Night Float & Attending $1 \& 4$ \\
\hline
\end{tabular}

FIG. 1. Changes in month-to-month resident service coverage following transition to a night float system. Arrows represent resident service transitions. Figure is available in color online only.

DRG 460 (spinal fusion except cervical without comorbidities/complications, $\left.\mathrm{R}^{2}=0.61, \mathrm{p}=0.04\right)$. We then ran a matched pairs Student t-test for the above DRG codes for the 2 years immediately prior to the initiation of night float, which was not statistically significant $(p=0.7)$. Thus, while the mean LOS for our most common DRGs had indeed trended down over a 7-year period, the effect had plateaued prior to initiation of the night float system and would not be expected to confound our results. Finally, we assessed the distribution of the 5 most common admission DRG codes for pre-night float and night float years, to confirm that the patients under study did not differ significantly between each year. The proportions of our 5 most common admission DRGs did not differ significantly between years (all p values $>0.4$ ).

\section{Length of ICU Stay}

The mean ICU LOS in the pre-night float group was 4.5 days (95\% CI 4.1-4.8 days), while the mean length of ICU stay in the night float group was 5.0 days $(95 \%$ CI 4.7-5.4 days). On bivariate analysis, there was a significant increase in ICU LOS after initiation of night float $(p=0.03)$. Adjusting for the increased number of service transitions in the night float era, there was no longer a significant difference in the ICU LOS after the institution of night float $\left(\mathrm{R}^{2}=0.17\right.$, root-mean-square error $=5.68, \mathrm{p}=$ 0.3). Again examining the subgroup of patients without a resident service transition, the effect was consistent. The mean ICU LOS in the pre-night float subgroup was 3.3 days (95\% CI 3.2-3.6), while the mean ICU LOS in the night float subgroup was 3.6 days (95\% CI 3.4-3.8). Bivariate analysis indicated that there was no significant difference in ICU LOS after the initiation of night float ( $p$ $=0.06$ ) among those patients who had not experienced a resident service transition.

Finally, we performed an additional matched pair analysis for 2 commonly performed procedures, cervical fusion and pituitary surgery. There were 34 matched pairs among patients undergoing cervical fusion. Among these patients, those treated prior to night float had no significant difference in overall hospital ( $p=0.30)$ or ICU ( $p=0.84)$ LOS relative to patients treated after initiation of night float. Among 24 matched pairs of patients undergoing pituitary surgery, there was again no significant difference in overall hospital $(p=0.79)$ or ICU $(p=0.90)$ LOS between cohorts.

\section{Mortality, Discharge Disposition, and 30-Day Readmission}

There were 62 deaths in the pre-night float group (2.9\%) and 53 deaths in the night float group (2.2\%). These groups were not significantly different $(\mathrm{p}=0.1)$, although the small number of events may have masked a statistically significant effect. Overall, $11.2 \%$ of patients were readmitted within 30 days during the 2-year study period. Exposure to the night float system was not associated with readmission rates $(\mathrm{p}=0.43)$ or discharge disposition $(\mathrm{p}=$ 0.45 ) on bivariate analysis.

\section{Resident Experience}

We found no significant difference between the num-

\section{Pre-Night Float}

\begin{tabular}{|c|c|c|c|c|c|c|c|}
\hline & Sunday & Monday & Tuesday & Wednesday & Thursday & Friday & Saturday \\
\hline Day & & & & & & & \\
\hline Night & & & & & & \\
\hline
\end{tabular}

\section{Night Float}

\begin{tabular}{|c|c|c|c|c|c|c|c|}
\hline & Sunday & Monday & Tuesday & Wednesday & Thursday & Friday & Saturday \\
\hline Day & & & & & & & \\
\hline Night & & & & & & \\
\hline
\end{tabular}

FIG. 2. Changes in day-to-day resident call structure following transition to a night float system. In the 24-hour resident call structure, call periods for each resident are represented as an individual color block for that resident. Figure is available in color online only. 
TABLE 2. Effect of a night float system on LOS and mortality

\begin{tabular}{clll}
\hline Analysis & Before Night Float $(n=2173)$ & After Night Float $(n=2446)$ & Comparison \\
\hline Bivariate analysis & & & \\
\hline LOS & 5.16 days $(95 \% \mathrm{Cl} 4.80-5.52)$ & 5.05 days $(95 \% \mathrm{Cl} 4.74-5.35)$ & $\mathrm{t}$-test: $p=0.65$ \\
\hline ICU LOS & 4.47 days $(95 \% \mathrm{Cl} 4.10-4.84)$ & 5.03 days $(95 \% \mathrm{Cl} 4.68-5.38)$ & $\mathrm{t}$-test: $p=0.03$ \\
\hline No. of deaths & $62(2.85 \%)$ & $53(2.17 \%)$ & $\mathrm{X}^{2}=2.23, p=0.14$ \\
\hline No. of handoffs & $253(11.6 \%)$ & $346(15.2 \%)$ & $\mathrm{X}^{2}=6.37, p=0.012$ \\
\hline Multivariate analysist & & & $\mathrm{p}=0.047$ \\
\hline LOS & LSM $=9.82(0.19$ SE $)$ & LSM $=9.41(0.18$ SE $)$ & $p=0.35$ \\
\hline ICU LOS & LSM $=6.58(0.19$ SE $)$ & LSM $=6.81(0.19$ SE $)$ & \\
\hline
\end{tabular}

$\mathrm{LSM}=$ least squares method.

* Continuous dependent variables were compared using 2-sample independent t-tests, while categorical dependent variables were compared using the chi-square test.

$\dagger$ Effect of night float on overall and ICU LOSs adjusted for resident service handoffs. Multivariate analysis was performed using ANCOVA.

ber of cases logged by PGY 2 residents between pre-night float and night float years (mean 140 cases pre-night float, mean 187 cases night float, $\mathrm{p}=0.17$ ). Additionally, there was no significant difference between cases logged by PGY 3 and 4 residents between pre-night float and night float years (mean 313 cases pre-night float, mean 323 cases night float, $\mathrm{p}=0.47$ ). PGY 5 was excluded as it consists of a dedicated research year at this institution, and PGY 6 was excluded as these residents do not take primary in-house call and would not be expected to experience any change in operative experience due to initiation of the night float system. Furthermore, there was no significant difference between duty hours reported among all residents on clinical services between pre-night float and night float years (mean 61.5 hours per week pre-night float, mean 64.4 hours per week night float, $p=0.39$ ). An online questionnaire was sent to the 19 residents at our institution for the 2014-2015 academic year. There was $100 \%$ survey response. Seven residents (37\%) had experience as the primary night float resident, and 18 (95\%) preferred the night float system to the previous 24-hour call system. Residents felt that the night float system improved adherence to duty hours, quality of life, time for independent study, patient outcomes, and overall residency training. Night float was felt to decrease resident fatigue and had no effect on resident case volume or educational opportunities. Full survey results are found in Table 3 and are demonstrated in Fig. 3.

\section{Discussion Historical Basis}

The initial impetus for instituting resident duty hour restrictions stemmed from a New York City grand jury finding that Libby Zion's death in 1984 resulted in part from resident fatigue. ${ }^{5}$ By 2001, federal legislation regulating resident work hours was drafted (HR3236 and S2614), leading to implementation of work hour restrictions in 2003 by the ACGME. Despite over a decade of national work hour restrictions, resident duty hours remain controversial, ${ }^{1,28}$ in part because of the scarcity of prospective trials comparing medical error rates between residents training under each system. Duty hour restrictions were primarily intended to reduce resident sleep deprivation, improve clinical performance, and thereby reduce medical errors. Improving resident quality of life and job satisfaction, and decreasing risk of motor vehicle crashes or needle stick injuries, were entirely secondary goals. ${ }^{15,27,30,31,35,42}$ Studies of the effect on patient outcomes have been decidedly mixed, with a recent meta-analysis showing negative effects of duty hour restrictions on patient outcomes and certification examinations for surgical residents. ${ }^{3}$ Multiple large national database retrospective studies have reported increased postoperative complications and hospital charges in neurosurgical patients following the implementation of duty hour restrictions, with no improvement in outcomes. ${ }^{7,8,14,21,32}$ While compelling, these studies are unable to parse out which factors accompanying work hour restrictions are responsible for the associated decline in outcomes. Decreased resident experience, increased frequen-

TABLE 3. Resident perspectives on a night float system

\begin{tabular}{lcc}
\hline \multicolumn{1}{c}{ Effects of night float on... } & Mean (SD)* & \multicolumn{1}{c}{ Likert Scale† } \\
\hline Resident case volume? & $2.84(0.96)$ & No effect \\
\hline $\begin{array}{l}\text { Educational opportunities/ } \\
\text { learning? }\end{array}$ & $3.47(0.77)$ & No effect \\
\hline Quality of life? & $4.11(0.74)$ & $\begin{array}{c}\text { Somewhat improves } \\
(p<0.01)\end{array}$ \\
\hline Continuity of care? & $3.58(1.02)$ & $\begin{array}{c}\text { Somewhat improves } \\
(p<0.01)\end{array}$ \\
\hline Adherence to duty hours? & $4.47(0.70)$ & $\begin{array}{c}\text { Somewhat improves } \\
(p<0.01)\end{array}$ \\
\hline Resident fatigue? & $4.00(0.67)$ & $\begin{array}{c}\text { Somewhat improves } \\
(p<0.01)\end{array}$ \\
\hline Time for independent study? & $3.74(0.73)$ & $\begin{array}{c}\text { Somewhat improves } \\
(p<0.01)\end{array}$ \\
\hline Patient outcomes (e.g., compli- & $3.53(0.70)$ & $\begin{array}{c}\text { Somewhat improves } \\
(p<0.01)\end{array}$ \\
\hline cations, LOS, mortality)? & $3.84(0.60)$ & $\begin{array}{c}\text { Somewhat improves } \\
(p<0.01)\end{array}$ \\
\hline Residency training overall & &
\end{tabular}

* Using a Likert Scale where 1 = significantly decreases/worsens; 2 = somewhat decreases/worsens; 3 = no effect; 4 = somewhat increases/improves; 5 = significantly increases/improves.

$\dagger p$ value shown reflects 1 -sample t-test comparing mean to a value of 3 (e.g., no effect). 


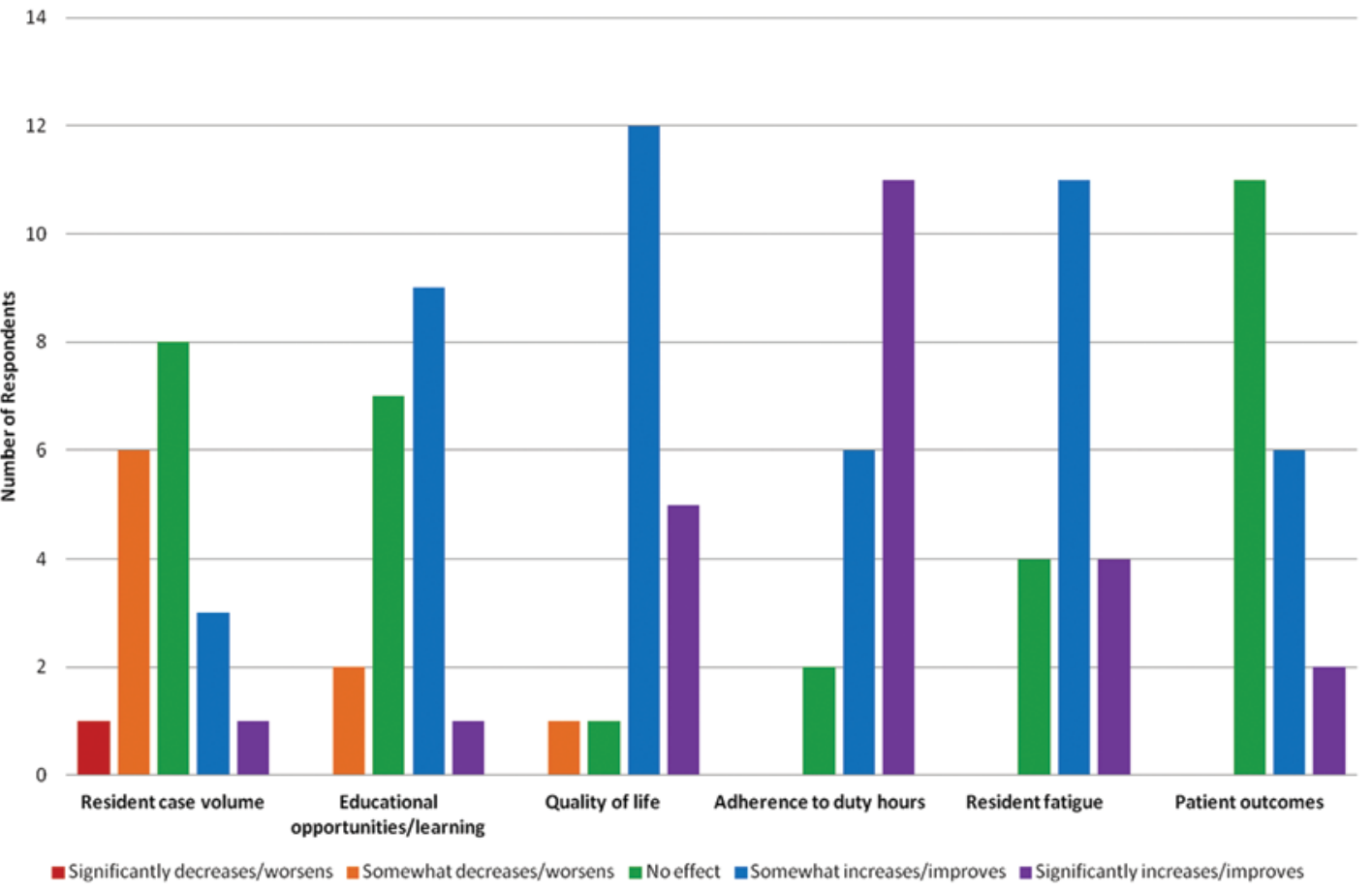

FIG. 3. Distribution of resident survey responses on implementation of night float. Figure is available in color online only.

cy of handoffs, and changes in billing and coding could all contribute to the observed difference in outcomes over this period.$^{41}$ Documentation of complications and medical errors is highly variable and prone to inaccuracy even in electronic medical records, and a recent large study of coding accuracy in surgical patients identified inaccuracy in $51 \%$ of patients, including changes in morbidity and incidence of complications. ${ }^{33}$ Within the neurosurgical literature, extant studies on complication rates following initiation of ACGME work hour restrictions use large administrative databases, which are subject to these inaccuracies. ${ }^{6-8,21}$ Furthermore, differences in patient outcomes between hospitals have been shown to result not from variations in complication rates, but rather from reduced speed and accuracy in identifying and responding to these complications. ${ }^{17,18}$ As a result, we attempted to limit our study to those outcomes that are reliable and uncontestable. We therefore posit that hospital LOS, ICU LOS, mortality, discharge disposition, and 30-day readmission rates may be a reasonable proxy for clinically significant complications. While work hour restrictions were a contributing factor in the decision to transition to a night float system at our institution, many factors are weighed in ultimately moving to night float coverage. Work hour restrictions predated the night float transition in this study, and thus would not be expected to directly influence our findings.

\section{Implication of Findings}

Rigorous evaluation of a night float system must account for effects on both patient outcomes and resident training. The primary goal of a night float system must be to promote high-quality patient care without sacrificing resident education, all while remaining within the bounds of the ACGME duty hour restrictions. High-quality studies have failed to convincingly demonstrate a positive impact of night float on patient outcomes, ${ }^{3}$ with several studies showing negative perceived effects of night float on patient safety., ${ }^{2,13,38}$ Studies on the influence of night float on resident education and wellness are similarly mixed, with both positive and negative effects having been demonstrated. .,13,15,19 $^{2}$

The stability of the UAB neurosurgical program was essential to demonstrating the impact of instituting the night float system. During the period under study, there were no significant changes in neurosurgical faculty, facilities, electronic medical record, hospital coverage, or patient population. To our knowledge, there have been no studies in the neurosurgical literature evaluating the relationship between night float and LOS, and as such this is a novel contribution. Importantly, coincident with the transition to a night float system was an increased frequency of resident service transitions, which were highly associated with LOS and ICU stay. In switching to a night float system, the resident rotation structure was altered to accommodate the new night float system. As a result, residents changed services more frequently. We demonstrate herein that, after adjusting for resident service transitions, institution of a night float system is associated with decreased hospital LOS among neurosurgical patients, with no observed correlation with ICU LOS, mortality, discharge disposition, or 30 -day readmission rates. The same effect was observed when considering only patients who did not experience a resident service transition.

It is essential to distinguish between resident service transitions - the change from one resident to another of a large number of patients for whom they have primary day-to-day responsibility-from the end-of-day handoff of care to a single resident providing care to all neurosurgi- 
cal patients overnight. Even under a 24-hour resident call system, there is a still a twice-daily handoff that occurs for all patients except those for whom the on-call resident holds primary responsibility. Additionally, due to ACGME work hour restrictions, even in a 24-hour call system the on-call resident must hand off responsibility for their patients for the postcall day. In a 24-hour call system, each resident will be on call every 3rd to 6th night. By contrast, in a night float system, the same resident typically provides overnight coverage 5-6 nights in a row. Therefore, the key difference with a night float system is that the resident providing overnight coverage is likely to be more familiar with all neurosurgical inpatients than a resident who is only on call every 4 th or 5th night. We hypothesize that this improved night-to-night continuity of care may be the driving force behind our findings.

Even in the absence of demonstrable errors in patient care, when a new resident assumes coverage of a large service, there is an inevitable lag period before the resident becomes knowledgeable about each patient and his or her potential barriers to discharge. Previous work demonstrated that resident service transitions were associated with increased LOS in neurosurgical patients at our institution. ${ }^{26}$ We suspect this adjustment period results in delays in patient discharge, with resultant unnecessary medical resource utilization. Seemingly minor factors such as prompt involvement of ancillary services such as physical therapy or social work may result in delays in discharge, without identifiable complications. In the bivariate analysis alone, night float appeared to be associated with increased ICU LOS, with no effect on overall LOS. Only after adjusting for resident service transitions was the night float system associated with reduced hospital LOS. A primary factor distinguishing our findings from prior studies is the inclusion of resident service transitions as a key confounding variable. This finding highlights a potential unintended consequence of switching to a night float system-an increased frequency of resident service transitions.

\section{Impact of Handoffs}

Provider handoffs are nuanced exchanges encompassing a wide range of subjective and objective clinical measures for the transition of patient care to another provider. ${ }^{12}$ Optimizing the handoff process has been evaluated in medical specialties, ${ }^{16,22}$ but scant attention has been paid to handoff effects in neurosurgery. ${ }^{6,36}$ Among medical residents, duty hour restrictions are associated with increased rates of self-reported medical errors, related to more handoffs. ${ }^{40}$ Discontinuity of care in any form contributes to medical complications and increased LOS, whether from handoffs, short versus long call period, or hospital stay encompassing a weekend. , $29,39,45,46$ In the surgical ICU, increased fragmentation of care through handoffs has been associated with higher rates of reintervention, ICU readmission, and complications. ${ }^{10}$ Resident handoffs are largely unstructured, have significant deficiencies, and frequently fail to convey essential patient information. ${ }^{43}$ High patient acuity, large censuses, and reliance on the inherently subjective nature of the neurological examination may render neurosurgical patients at particular risk for handoff-related errors. ${ }^{44}$ Standardized tools, deliberate practice, and mini- mizing distractions may be used to improve the quality of resident handoffs. ${ }^{4}$

\section{Night Float and Resident Training}

Most general surgery residents perceive that systemic flaws are responsible for the majority of medical errors, and that further work hour restrictions will not further improve medical care. ${ }^{11}$ An abundance of caution must be exercised when considering the transition to night float, so as not to diffuse patient ownership, limit operative experience, and increase noneducational activities. Residents in our program take a nearly universally positive stance toward the night float system, citing improved adherence to duty hours, quality of life, time for independent study, patient outcomes, and overall residency training. Night float was felt to decrease resident fatigue and have no effect on resident case volume or educational opportunities. At our institution, under the 24-hour call system, the on-call resident was unable to reliably participate in operative cases during the day, and, due to ACGME work hour restrictions, was unable to participate in operative cases on the postcall day. However, no studies to date have formally evaluated the effect of night float on operative case volume in neurosurgery, and data from other surgical disciplines are mixed. ${ }^{25,37}$ While we found no significant difference in resident reported duty hours or number of operative cases logged, the small number of residents involved limits the generalizability of our findings. Future investigations into the effect of implementing a night float system must consider patient-centered outcomes as well as metrics of resident training.

\section{Limitations}

Limitations of this study include retrospective data collection, lack of long-term outcomes or specific complication rates, and performance at a single institution. Length of stay was initially selected as our primary outcome of interest because it is uncontestable and can be considered a proxy for significant complications. It is not known if the results from our study are generalizable to other neurosurgery residency programs. There are likely modest regional variations in patient populations and composition of neurosurgical services. There is also variation between institutions on how resident coverage is distributed and assignment of responsibilities of residents and advanced practitioners. Finally, we did not include patients for whom neurosurgery was a consulting service, including polytrauma patients with neurological injuries. Length of stay for these patients is dictated by a multitude of factors for which it would be difficult to control, and as such only patients for whom a neurosurgeon was the primary physician were included. For these reasons, our findings warrant validation by future studies.

\section{Conclusions}

Transitioning to a resident night float system increases the number of resident service transitions, the inevitable sequelae of which includes a heightened risk of medical errors. Previous studies have focused on the deleterious influence of duty-hour restrictions, with the premise that 
resident education has declined in the post- 2003 era, with patient outcomes suffering as a result. We find that transitioning to the night float system increased ICU LOS with no effect on hospital LOS, and only by adjusting for resident service transitions do we observe decreased overall hospital LOS, with no effect on ICU LOS.

Patient mortality, discharge disposition, and readmission rates were not impacted by the institution of the night float system in our population. Furthermore, resident perception of the night float system was that it was positive for patient care, resident training, and decreasing resident fatigue, while resident duty hours and operative cases logged were unaffected. This study supports the argument that increased frequency of service transitions, not poor quality of resident training, may be the cause of poorer outcomes among neurosurgical patients in the modern era of training. Prospective cohort studies are needed to definitively establish associations between night float and patient outcomes. Futures studies should also prospectively analyze means of improving continuity of care, enhancing the night float system, and mitigating the effect of resident service transitions on patient outcomes.

\section{Acknowledgments}

Dr. Davis and Dr. Kuhn completed this work as Women's Leadership Council Clinical Scholars in the Department of Neurosurgery at the University of Alabama at Birmingham.

\section{References}

1. Abraham T, Freitas M, Frangos S, Frankel HL, Rabinovici R: Are resident work-hour limitations beneficial to the trauma profession? Am Surg 72:35-41, 2006

2. Accreditation Council for Graduate Medical Education: The ACGME 2011 Duty Hour Standard. Chicago: ACGME, 2011 (https://www.acgme.org/Portals/0/PDFs/jgme-monograph[1]. pdf) [Accessed May 27, 2016]

3. Ahmed N, Devitt KS, Keshet I, Spicer J, Imrie K, Feldman L, et al: A systematic review of the effects of resident duty hour restrictions in surgery: impact on resident wellness, training, and patient outcomes. Ann Surg 259:1041-1053, 2014

4. Anderson CE, Nicksa GA, Stewart L: Distractio.ns during resident handoffs: incidence, sources, and influence on handoff quality and effectiveness. JAMA Surg 150:396-401, 2015

5. Asch DA, Parker RM: The Libby Zion case. One step forward or two steps backward? N Engl J Med 318:771-775, 1988

6. Babu MA, Nahed BV, Heary RF: Investigating the scope of resident patient care handoffs within neurosurgery. PLoS One 7:e41810, 2012

7. Babu R, Thomas S, Hazzard MA, Friedman AH, Sampson $\mathrm{JH}$, Adamson C, et al: Worse outcomes for patients undergoing brain tumor and cerebrovascular procedures following the ACGME resident duty-hour restrictions. J Neurosurg 121:262-276, 2014

8. Babu R, Thomas S, Hazzard MA, Lokhnygina YV, Friedman AH, Gottfried ON, et al: Morbidity, mortality, and health care costs for patients undergoing spine surgery following the ACGME resident duty-hour reform: Clinical article. J Neurosurg Spine 21:502-515, 2014

9. Blecker S, Shine D, Park N, Goldfeld K, Scott Braithwaite R, Radford MJ, et al: Association of weekend continuity of care with hospital length of stay. Int J Qual Health Care 26:530-537, 2014
10. Bollschweiler E, Krings A, Fuchs KH, Pistorius G, Bein T, Otto U, et al: Alternative shift models and the quality of patient care. An empirical study in surgical intensive care units. Langenbecks Arch Surg 386:104-109, 2001

11. Borman KR, Jones AT, Shea JA: Duty hours, quality of care, and patient safety: general surgery resident perceptions. J Am Coll Surg 215:70-79, 2012

12. Burton MC, Kashiwagi DT, Kirkland LL, Manning D, Varkey P: Gaining efficiency and satisfaction in the handoff process. J Hosp Med 5:547-552, 2010

13. Desai SV, Feldman L, Brown L, Dezube R, Yeh HC, Punjabi N, et al: Effect of the 2011 vs 2003 duty hour regulationcompliant models on sleep duration, trainee education, and continuity of patient care among internal medicine house staff: a randomized trial. JAMA Intern Med 173:649-655, 2013

14. Dumont TM, Rughani AI, Penar PL, Horgan MA, Tranmer BI, Jewell RP: Increased rate of complications on a neurological surgery service after implementation of the Accreditation Council for Graduate Medical Education work-hour restriction. J Neurosurg 116:483-486, 2012

15. Fletcher KE, Underwood W III, Davis SQ, Mangrulkar RS, McMahon LF Jr, Saint S: Effects of work hour reduction on residents' lives: a systematic review. JAMA 294:1088-1100, 2005

16. Gakhar B, Spencer AL: Using direct observation, formal evaluation, and an interactive curriculum to improve the sign-out practices of internal medicine interns. Acad Med 85:1182-1188, 2010

17. Ghaferi AA, Birkmeyer JD, Dimick JB: Complications, failure to rescue, and mortality with major inpatient surgery in Medicare patients. Ann Surg 250:1029-1034, 2009

18. Ghaferi AA, Birkmeyer JD, Dimick JB: Variation in hospital mortality associated with inpatient surgery. N Engl J Med 361:1368-1375, 2009

19. Goldstein MJ, Kim E, Widmann WD, Hardy MA: A 360 degrees evaluation of a night-float system for general surgery: a response to mandated work-hours reduction. Curr Surg 61:445-451, 2004

20. Grady MS, Batjer HH, Dacey RG: Resident duty hour regulation and patient safety: establishing a balance between concerns about resident fatigue and adequate training in neurosurgery. J Neurosurg 110:828-836, 2009

21. Hoh BL, Neal DW, Kleinhenz DT, Hoh DJ, Mocco J, Barker FG II: Higher complications and no improvement in mortality in the ACGME resident duty-hour restriction era: an analysis of more than 107,000 neurosurgical trauma patients in the Nationwide Inpatient Sample database. Neurosurgery 70:1369-1382, 2012

22. Hsiao AL, Shiffman RN: Dropping the baton during the handoff from emergency department to primary care: pediatric asthma continuity errors. Jt Comm J Qual Patient Saf 35:467-474, 2009

23. Jagannathan J, Vates GE, Pouratian N, Sheehan JP, Patrie J, Grady MS, et al: Impact of the Accreditation Council for Graduate Medical Education work-hour regulations on neurosurgical resident education and productivity. J Neurosurg 110:820-827, 2009

24. Jagsi R, Kitch BT, Weinstein DF, Campbell EG, Hutter M, Weissman JS: Residents report on adverse events and their causes. Arch Intern Med 165:2607-2613, 2005

25. Kelly RJ Jr, Senkowski CK: Effect of the night float system on operative case volume for senior surgical residents. J Surg Educ 66:314-318, 2009

26. Kuhn EN, Davis MC, Agee BS, Oster RA, Markert JM: Effect of resident handoffs on length of hospital and intensive care unit stay in a neurosurgical population: a cohort study. J Neurosurg [epub ahead of print December 18, 2015; DOI: 10.3171/2015.7.JNS15920] 
27. Leonard C, Fanning N, Attwood J, Buckley M: The effect of fatigue, sleep deprivation and onerous working hours on the physical and mental wellbeing of pre-registration house officers. Ir J Med Sci 167:22-25, 1998

28. Lewis FR: Comment of the American Board of Surgery on the recommendations of the Institute of Medicine report, "Resident Duty Hours: Enhancing Sleep, Supervision, and Safety." Surgery 146:410-419, 2009

29. Lofgren RP, Gottlieb D, Williams RA, Rich EC: Post-call transfer of resident responsibility: its effect on patient care. $\mathbf{J}$ Gen Intern Med 5:501-505, 1990

30. Makary MA, Al-Attar A, Holzmueller CG, Sexton JB, Syin $\mathrm{D}$, Gilson MM, et al: Needlestick injuries among surgeons in training. N Engl J Med 356:2693-2699, 2007

31. Marcus CL, Loughlin GM: Effect of sleep deprivation on driving safety in housestaff. Sleep 19:763-766, 1996

32. Norby K, Siddiq F, Adil MM, Haines SJ: The effect of duty hour regulations on outcomes of neurological surgery in training hospitals in the United States: duty hour regulations and patient outcomes. J Neurosurg 121:247-261, 2014

33. Nouraei SA, Hudovsky A, Frampton AE, Mufti U, White NB, Wathen CG, et al: A study of clinical coding accuracy in surgery: implications for the use of administrative big data for outcomes management. Ann Surg 261:1096-1107, 2015

34. Oshimura JM, Sperring J, Bauer BD, Carroll AE, Rauch DA: Changes in inpatient staffing following implementation of new residency work hours. J Hosp Med 9:640-645, 2014

35. Philibert I: Sleep loss and performance in residents and nonphysicians: a meta-analytic examination. Sleep 28:13921402, 2005

36. Riesenberg LA, Leitzsch J, Massucci JL, Jaeger J, Rosenfeld JC, Patow C, et al: Residents' and attending physicians' handoffs: a systematic review of the literature. Acad Med 84:1775-1787, 2009

37. Scally CP, Reames BN, Teman NR, Fritze DM, Minter RM, Gauger PG: Preserving operative volume in the setting of the 2011 ACGME duty hour regulations. J Surg Educ 71:580 586,2014

38. Schenarts P, Bowen J, Bard M, Sagraves S, Toschlog E, Goettler $\mathrm{C}$, et al: The effect of a rotating night-float coverage scheme on preventable and potentially preventable morbidity at a level 1 trauma center. Am J Surg 190:147-152, 2005

39. Schuberth JL, Elasy TA, Butler J, Greevy R, Speroff T, Dittus RS, et al: Effect of short call admission on length of stay and quality of care for acute decompensated heart failure. Circulation 117:2637-2644, 2008

40. Sen S, Kranzler HR, Didwania AK, Schwartz AC, Amarnath
S, Kolars JC, et al: Effects of the 2011 duty hour reforms on interns and their patients: a prospective longitudinal cohort study. JAMA Intern Med 173:657-663, 2013

41. Simien C, Holt KD, Richter TH: The impact of ACGME work-hour reforms on the operative experience of fellows in surgical subspecialty programs. J Grad Med Educ 3:111117,2011

42. Stamp T, Termuhlen P, Miller S, Nolan D, Hutzel P, Gilchrist $\mathrm{J}$, et al: Before and after resident work hour limitations: an objective assessment of the well-being of surgical residents. Curr Surg 62:117-121, 2005

43. Tapia NM, Fallon SC, Brandt ML, Scott BG, Suliburk JW: Assessment and standardization of resident handoff practices: PACT project. J Surg Res 184:71-77, 2013

44. Thaller M, Hughes T: Inter-rater agreement of observable and elicitable neurological signs. Clin Med (Lond) 14:264-267, 2014

45. Van Eaton EG, Horvath KD, Lober WB, Rossini AJ, Pellegrini CA: A randomized, controlled trial evaluating the impact of a computerized rounding and sign-out system on continuity of care and resident work hours. J Am Coll Surg 200:538-545, 2005

46. Yeung L, Miraflor E, Garcia A, Victorino GP: Effect of surgery resident change of shift on trauma resuscitations and outcomes. J Surg Educ 70:87-94, 2013

\section{Disclosures}

The authors report no conflict of interest concerning the materials or methods used in this study or the findings specified in this paper.

\section{Author Contributions}

Conception and design: Davis, Markert. Acquisition of data: Davis, Kuhn. Analysis and interpretation of data: Davis, Kuhn. Drafting the article: Davis, Kuhn. Critically revising the article: Davis, Kuhn. Reviewed submitted version of manuscript: all authors. Approved the final version of the manuscript on behalf of all authors: Davis. Statistical analysis: Davis, Kuhn, Agee, Oster. Study supervision: Markert.

\section{Correspondence}

Matthew C. Davis, Department of Neurosurgery, University of Alabama at Birmingham, 1720 Second Ave. South, FOT 1062. Birmingham, AL 35294-3410. email: matthewdavis@uabmc.edu. 